\title{
MICZ-Kepler problems in all dimensions
}

\author{
Guowu Meng ${ }^{\text {a) }}$ \\ Department of Mathematics, Hong Kong University of Science and Technology, \\ Clear Water Bay, Kowloon, Hong Kong
}

(Received 18 July 2006; accepted 5 February 2007; published online 20 March 2007)

The Kepler problem is a physical problem about two bodies which attract each other by a force proportional to the inverse square of the distance. The MICZKepler problems are its natural cousins and have been previously generalized from dimension 3 to dimension 5. In this paper, we construct and analyze the (quantum) MICZ-Kepler problems in all dimensions higher than 2. () 2007 American Institute of Physics. [DOI: 10.1063/1.2712420]

\section{INTRODUCTION}

The Kepler problem is the physics problem about two bodies which attract each other by a force proportional to the inverse square of the distance. By solving this problem in classical mechanics, Newton gave a satisfactory explanation for Kepler's laws for the planetary motion. The Kepler problem plays a significant role in the development of quantum mechanics, too; in fact, the solution of this problem in the Schrödinger wave mechanics firmly puts the Schrödinger equation right at the center of quantum mechanics.

After more than three centuries, the Kepler problem still plays an important role in mathematics and physics. There has been a continuous interest in this problem; in particular, in the last three decades we have witnessed an explosion of its interactions with quantum mechanics, celestial mechanics, and mathematics. For a recent comprehensive treatment of the Kepler problem, the interested readers may consult Ref. 1.

The MICZ-Kepler problems are natural cousins of the Kepler problem, and they were independently discovered by McIntosh and Cisneros ${ }^{2}$ and Zwanziger ${ }^{3}$ more than 30 years ago. Roughly speaking, a MICZ-Kepler problem is the Kepler problem in the case when the nucleus of a hypothetic hydrogen atom also carries a magnetic charge. These generalized problems share the following characteristic beauty with the Kepler problem: the existence of the Runge-Lenz vector and the dynamical spin (4) symmetry for the bound states; therefore, they provide a rich family of examples for the exploration of the extra hidden dynamic symmetry.

The Hamiltonian of a MICZ-Kepler problem is constructed from that of the Kepler problem by adding the vector potential of a Dirac monopole and a repulsive centrifugal potential; explicitly, we have

$$
H=\frac{1}{2 m}(\mathbf{p}+e \mathbf{A})^{2}+\frac{\mu^{2}}{2 m r^{2}}-\frac{e^{2}}{r},
$$

where $\mathbf{p}$ is the canonical momentum of the electron, $\mathbf{A}$ is the vector potential of a Dirac monopole, $r$ is the distance from the electron to the hydrogen nucleus, $m$ is the (reduced) mass of the electron, $e$ is the fundamental unit of the electric charge, and $\mu$ is the magnetic charge of the Dirac monopole measured in unit of $c / e$, i.e., $\mu(c / e)$ is the magnetic charge of the Dirac monopole, here $c$ is the speed of light in vacuum (the Dirac quantization condition becomes $\mu / \hbar=$ a half-integer).

Quantum mechanically, via rescaling

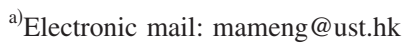




$$
r \rightarrow \frac{\hbar^{2}}{m e^{2}} r, \quad \mu \rightarrow \hbar \mu,
$$

we arrive at the following Hamiltonian operator:

$$
\hat{H}=\frac{m e^{4}}{\hbar^{2}}\left(-\frac{1}{2} \Delta_{A}+\frac{\mu^{2}}{2 r^{2}}-\frac{1}{r}\right):=\frac{m e^{4}}{\hbar^{2}} \hat{h},
$$

where

$$
\hat{h}=-\frac{1}{2} \Delta_{A}+\frac{\mu^{2}}{2 r^{2}}-\frac{1}{r} .
$$

[We remark that $\hat{h}$ is dimensionless and is expressed in terms of dimensionless quantities. It is $\hat{h}$ (not $\hat{H}$ ) that will be generalized later.] Here $\Delta_{A}$ is the Laplace operator twisted by the gauge potential $A$ of a Dirac monopole, and $\mu=0, \pm \frac{1}{2}, \pm 1, \ldots$ is the magnetic charge of the Dirac monopole measured in terms of the fundamental unit (the case $\mu=0$ corresponds to the Kepler problem). Locally, with a gauge chosen, we have

$$
\Delta_{A}=\nabla_{a} \nabla_{a}
$$

where the repeated index $a$ is summed up. Here $\nabla_{a}$ is the $a$ th covariant partial derivative and is written as $\partial_{a}+i A_{a}$ by physicists with $\mathbf{A}=\left(A_{1}, A_{2}, A_{3}\right)$ being the gauge potential of the Dirac magnetic monopole. Mathematically $\nabla_{a}=\partial_{a}+\omega_{a}$ where $\omega=\omega_{a} \mathrm{~d} x_{a}$ has been previously identified with the Levi-Civita spin connection form of the cylindrical metric

$$
\mathrm{d} s^{2}=\frac{1}{r^{2}}\left(\mathrm{~d} x_{1}^{2}+\mathrm{d} x_{2}^{2}+\mathrm{d} x_{3}^{2}\right)
$$

on the punctured three space; see Ref. 4 for the details.

The MICZ-Kepler problems exist in higher dimensions just as the Kepler problem does, and that is a main observation here. In fact, the existence in dimension 5 has been previously observed, ${ }^{5}$ however, the existence in all dimensions greater than 2 , though very straightforward from a canonical geometric point of view, was probably not expected by the community. This overlook is very likely due to a general belief in the literature: the existence of Dirac monopoles and its five-dimensional analog (the Yang monopoles ${ }^{6}$ ) has to do with the existence of the division algebras or Hopf bundles.

I would like to remark that the Runge-Lenz vector extends naturally and is conserved in odd dimensions, however, it is not conserved in even dimensions unless $\mu=0$ or $\frac{1}{2}$. As a consequence, although they will be shown to be always integrable, the quantum MICZ-Kepler problems are shown here to be superintegrable only when either the dimension is odd or $\mu=0, \frac{1}{2}$.

In Sec. II, we construct the MICZ-Kepler problems in all dimensions and then state the main results. The construction is geometric and canonical. A key ingredient in the construction is the higher dimensional generalization of the Dirac monopoles-a canonical geometric object that has been used in Ref. 7. In Sec. III, we first introduce the explicit formulas for the gauge potential of these generalized Dirac monopoles; then we list and prove some crucial identities necessary for the exhibition of the extra large hidden dynamical symmetry. In Sec. IV, we introduce the angular momentum and Rung-Lenz vector for our MICZ-Kepler problems and derive the symmetry algebra in the case when either the dimension is odd or $\mu=0, \frac{1}{2}$. In Sec. V, we obtain the energy spectrum and the energy eigenspaces for bound states by using Painlevé analysis plus representation theory, and then show that the Hilbert space of bound states has a hidden dynamical $\operatorname{spin}(D+1)$ symmetry for a $D$-dimensional MICZ-Kepler problem even though in general the Runge-Lenz vector fails to be conserved when $D$ is even.

We remark that the MICZ-Kepler problems in higher dimensions constructed here are based on modern geometry, but they are solved by classical analytic method with the help of the 
representation theory for Lie groups. The solution of these new MICZ-Kepler problems can in principle be solved by the modern geometric quantization approach pioneered by $\mathrm{Simms}^{8}$ and Mladenov and Tsanov, ${ }^{9,10}$ but that will be reserved for the future for the following reasons: (1) the primary objective of this paper is to inform the experts in the fields that the MICZ-Kepler problems do exist in higher dimensions, (2) the classical analytic approach is more elementary and easier to understand, and (3) the modern geometric quantization approach is a bit more involved and deserves an independent research.

\section{THE MAIN RESULTS}

From the physics point of view, a MICZ-Kepler problem is obtained from the Kepler problem by adding a suitable background magnetic field while at the same time making a suitable adjustment to the scalar Coulomb potential so that the problem is still integrable. The background magnetic field is just the spin connection of the cylindrical metric on the configuration space that we have mentioned in the Introduction. [For readers without sufficient background in modern geometry, just take our explicit formulas for gauge potential in Eq. (5) for granted.] The configuration space is the punctured Euclidean space. With this in mind, we are now ready to give the detailed presentation of our generalized MICZ-Kepler problems.

Let $D \geqslant 3$ be an integer and $\mathbb{R}_{*}^{D}$ be the punctured $D$ space, i.e., $\mathbb{R}^{D}$ with the origin removed. Let $\mathrm{d} s^{2}$ be the cylindrical metric on $\mathbb{R}_{*}^{D}$. Then $\left(\mathbb{R}_{*}^{D}, \mathrm{~d} s^{2}\right)$ is the product of the straight line $\mathbb{R}$ with the round sphere $S^{D-1}$. When $D$ is odd, we let $\mathcal{S}_{ \pm}$be the positive/negative spinor bundle of $\left(\mathbb{R}_{*}^{D}, \mathrm{~d} s^{2}\right)$, and when $D$ is even, we let $\mathcal{S}$ be the spinor bundle of $\left(\mathbb{R}_{*}^{D}, \mathrm{~d} s^{2}\right)$. Note that these bundles correspond to the fundamental spin representations $\mathbf{s}_{ \pm}$of so(even) and $\mathbf{s}$ of so(odd), respectively.

The above spinor bundles come with a natural $\mathrm{SO}(D)$ invariant connection-the Levi-Civita spin connection of $\left(\mathrm{R}_{*}^{D}, \mathrm{~d} s^{2}\right)$. As a result, the Young products of $I$ copies of these bundles, denoted by $\mathcal{S}_{+}^{I}, \mathcal{S}_{-}^{I}$ (when $D$ is odd) and $\mathcal{S}^{I}$ (when $D$ is even), respectively, come with a natural connection, too.

For the sake of notational sanity, from here on, when $D$ is odd and $\mu$ is a half-integer, we rewrite $\mathcal{S}_{+}^{2 \mu}$ as $\mathcal{S}^{2 \mu}$ if $\mu \geqslant 0$ and rewrite $\mathcal{S}_{-}^{-2 \mu}$ as $\mathcal{S}^{2 \mu}$ if $\mu \leqslant 0$; moreover, we adopt this convention for $\mu=0$ : $\mathcal{S}^{0}$ is the product complex line bundle with the product connection. Note that $\mathcal{S}^{2 \mu}$ is our analog of the Dirac monopole with magnetic charge $\mu$, and its corresponding representation of so $(D-1)$ will be denoted by $\mathbf{s}^{2 \mu}$.

We are now ready to present our definitions.

Definition 1: Let $n \geqslant 1$ be an integer and $\mu$ a half-integer. The $(2 n+1)$-dimensional MICZKepler problem labeled by $\mu$ is defined to be the quantum mechanical system on $\mathbb{R}_{*}^{2 n+1}$ for which the wave functions are sections of $\mathcal{S}^{2 \mu}$, and the Hamiltonian is

$$
\hat{h}=-\frac{1}{2} \Delta_{\mu}+\frac{(n-1)|\mu|+\mu^{2}}{2 r^{2}}-\frac{1}{r},
$$

where $\Delta_{\mu}$ is the Laplace operator twisted by $\mathcal{S}^{2 \mu}$.

Definition 2: Let $n>1$ be an integer and $\mu \geqslant 0$ a half-integer. The $2 n$-dimensional MICZKepler problem is defined to be the quantum mechanical system on $\mathbb{R}_{*}^{2 n}$ for which the wave functions are sections of $\mathcal{S}^{2 \mu}$, and the Hamiltonian is

$$
\hat{h}=-\frac{1}{2} \Delta_{\mu}+\frac{(n-1) \mu}{2 r^{2}}-\frac{1}{r},
$$

where $\Delta_{\mu}$ is the Laplace operator twisted by $\mathcal{S}^{2 \mu}$.

We remark that, upon a choice of a local gauge, the background magnetic potential $A_{\alpha}$ can be explicitly written down, then $\Delta_{\mu}=\Sigma_{\alpha}\left(\partial_{\alpha}+i A_{\alpha}\right)^{2}$ can be explicitly written down, too. We are now ready to state our main results.

Theorem 1:For the $(2 n+1)$-dimensional MICZ-Kepler problem labeled by $\mu$, the following statements are true: 
(1) The negative-energy spectrum is

$$
E_{I}=-\frac{1 / 2}{(I+n+|\mu|)^{2}},
$$

where $I=0,1,2, \ldots$.

(2) The Hilbert space $\mathcal{H}$ of negative-energy states admits a linear $\operatorname{spin}(2 n+2)$ action under which there is a decomposition

$$
\mathcal{H}=\oplus_{I=0}^{\infty} \mathcal{H}_{I},
$$

where $\mathcal{H}_{I}$ is a model for the irreducible $\operatorname{spin}(2 n+2)$ representation whose highest weight is $(I+|\mu|,|\mu|, \ldots,|\mu|, \mu)$.

(3) $\operatorname{spin}(2 n+1,1)$ acts linearly on the positive-energy states and $\operatorname{spin}(2 n+1) \rtimes \mathbb{R}^{2 n+1}$ acts linearly on the zero-energy states.

(4) The linear action in either part (2) or part (3) extends the manifest linear action of $\operatorname{spin}(2 n+1)$, and $\mathcal{H}_{I}$ in part (2) is the energy eigenspace with eigenvalue $E_{I}$ in part (1).

Theorem 2:For the 2n-dimensional MICZ-Kepler problem labeled by $\mu$, the following statements are true:

(1) The negative-energy spectrum is

$$
E_{I}=-\frac{1 / 2}{(I+n+\mu-1 / 2)^{2}},
$$

where $I=0,1,2, \ldots$.

(2) The Hilbert space $\mathcal{H}$ of negative-energy states admits a linear $\operatorname{spin}(2 n+1)$ action under which there is a decomposition

$$
\mathcal{H}=\oplus_{I=0}^{\infty} \mathcal{H}_{I},
$$

where $\mathcal{H}_{I}$ is a model for the irreducible $\operatorname{spin}(2 n+1)$ representation whose highest weight is $(I+\mu, \mu, \ldots, \mu)$.

(3) When $\mu=0$ or $\mu=\frac{1}{2}, \operatorname{spin}(2 n, 1)$ acts linearly on the positive-energy states and $\operatorname{spin}(2 n) \rtimes \mathbb{R}^{2 n}$ acts linearly on the zero-energy states;

(4) The linear action in part (2) extends the manifest linear action of $\operatorname{spin}(2 n)$, and $\mathcal{H}_{I}$ in part (2) is the energy eigenspace with eigenvalue $E_{I}$ in part (1).

We remark that, based on the analysis done in later sections, we know that bound states are always the ones with negative-energy eigenvalues.

\section{GENERALIZED DIRAC MONOPOLES}

We write $\mathbf{r}=\left(x_{1}, x_{2}, \ldots, x_{D-1}, x_{0}\right)$ for a point in $\mathbb{R}^{D}$ and $r$ for the length of $\mathbf{r}$. The small Greek letters $\mu, \nu$, etc., run from 0 to $D-1$ and the small Latin letters $a, b$, etc., run from 1 to $D-1$. We use the Einstein convention: the repeated index is always summed up.

To do computations, we just need to choose a gauge on $\mathrm{R}^{D}$ minus the negative 0 th axis and then write down the gauge potential explicitly. We have done that before in Eq. (10) of Ref. 4. Note that, if we use the rectangular coordinates $\mathbf{r}=\left(\mathbf{x}, x_{0}\right)$, then the gauge potential $A=A_{\mu} \mathrm{d} x_{\mu}$ from Eq. (10) of Ref. 4 can be written as

$$
A_{0}=0, \quad A_{b}=-\frac{1}{r\left(r+x_{0}\right)} x_{a} \gamma_{a b},
$$

where $\gamma_{a b}=(i / 4)\left[\gamma_{a}, \gamma_{b}\right]$ with $\gamma_{a}$ the "gamma matrix" for physicists. (In Ref. 4 we only consider the case that $D$ is odd, the topological nontrivial case, but the basic construction there is valid in any dimension, see Appendix $\mathrm{A}$ in Ref. 7.) Note that $\gamma_{a}=i e_{a}$ with $e_{a}$ being the element in the 
Clifford algebra that corresponds to the $a$ th standard coordinate vector of $\mathrm{R}^{D-1}$.

It is straightforward to calculate the gauge field strength $F_{\mu \nu}=\partial_{\mu} A_{\nu}-\partial_{\nu} A_{\mu}+i\left[A_{\mu}, A_{\nu}\right]$ and get

$$
\begin{gathered}
F_{0 b}=\frac{1}{r^{3}} x_{a} \gamma_{a b}, \\
F_{a b}=-\frac{2 \gamma_{a b}}{r\left(r+x_{0}\right)}+\frac{1}{r^{2}\left(r+x_{0}\right)^{2}} \times\left(\left(2+\frac{x_{0}}{r}\right) x_{c}\left(x_{a} \gamma_{c b}-x_{b} \gamma_{c a}\right)+i x_{d} x_{c}\left[\gamma_{d a}, \gamma_{c b}\right]\right)
\end{gathered}
$$

The following lemma is crucially used when we check the dynamical symmetry of our models.

Lemma 1: For the gauge potential defined in Eq. (5), we have the following.

(1) Let $\nabla_{\alpha}=\partial_{\alpha}+i A_{\alpha}$; then the following identities are valid in any representation:

$$
\begin{gathered}
F_{\mu \nu} F^{\mu \nu}=\frac{2}{r^{4}} c_{2} \text { where } c_{2}=c_{2}[\operatorname{so}(D-1)]=\frac{1}{2} \gamma_{a b} \gamma_{a b} \\
{\left[\nabla_{\kappa}, F_{\mu \nu}\right]=\frac{1}{r^{2}}\left(x_{\mu} F_{\nu \kappa}+x_{\nu} F_{\kappa \mu}-2 x_{\kappa} F_{\mu \nu}\right)} \\
x_{\mu} A_{\mu}=0, \quad x_{\mu} F_{\mu \nu}=0,\left[\nabla_{\mu}, \quad F_{\mu \nu}\right]=0 \\
r^{2}\left[F_{\mu \nu}, F_{\alpha \beta}\right]+i F_{\mu \beta} \delta_{\alpha \nu}-i F_{\nu \beta} \delta_{\alpha \mu}+i F_{\alpha \mu} \delta_{\beta \nu}-i F_{\alpha \nu} \delta_{\beta \mu}=\frac{i}{r^{2}}\left(x_{\mu} x_{\alpha} F_{\beta \nu}+x_{\mu} x_{\beta} F_{\nu \alpha}-x_{\nu} x_{\alpha} F_{\beta \mu}\right. \\
\left.-x_{\nu} x_{\beta} F_{\mu \alpha}\right) .
\end{gathered}
$$

(2) When $D=2 n+1$, the identity

$$
r^{2} F_{\lambda \alpha} F_{\lambda \beta}=\frac{c_{2}}{n}\left(\frac{1}{r^{2}} \delta_{\alpha \beta}-\frac{x_{\alpha} x_{\beta}}{r^{4}}\right)+i(n-1) F_{\alpha \beta}
$$

holds in the irreducible representation $\mathrm{s}^{2 \mu}$ of $\mathrm{so}(2 n)$ whose highest weight is of the form $(|\mu|, \ldots,|\mu|, \mu)$.

(3) When $D=2 n$, the identity

$$
r^{2} F_{\lambda \alpha} F_{\lambda \beta}=\frac{n-1}{2}\left(\frac{1}{r^{2}} \delta_{\alpha \beta}-\frac{x_{\alpha} x_{\beta}}{r^{4}}\right)+i\left(n-\frac{3}{2}\right) F_{\alpha \beta}
$$

holds in the fundamental spin representation $\mathbf{s}$ of $\operatorname{so}(2 n-1)$.

\section{A. Proof of Lemma 1}

The verification of these identities is just a direct and lengthy calculation. However, if we exploit the symmetry, we just need to check the identities at point $\mathbf{r}_{0}=(0, \ldots, 0, r)$, a much easier task. For example, since

$$
A_{\mu}=0, \quad F_{0 a}=0, \quad F_{a b}=-\frac{1}{r^{2}} \gamma_{a b}
$$

at $\mathbf{r}_{0}$, identity (8) is obvious.

Proof of part (1): We have just remarked that identity (8) is obvious. Also, 


$$
x_{\mu} F_{\mu \nu}\left|\mathbf{r}_{0}=x_{0} F_{0 \nu}\right| \mathbf{r}_{0}=0 .
$$

It is also easy to see that $\left.x_{\mu} A_{\mu}\right|_{\mathbf{r}_{0}}=0$ and

$$
\left.\left[\nabla_{\mu}, F_{\mu \nu}\right]\right|_{\mathbf{r}_{0}}=\left.\partial_{\mu} F_{\mu \nu}\right|_{\mathbf{r}_{0}}=0 .
$$

Therefore, identity (11) is checked.

To check identity (10), first we assume that $\mu=0$ and $\nu=b$, then we need to check that

$$
\partial_{\kappa} F_{0 b}=\frac{1}{r} F_{b \kappa}
$$

at $\mathbf{r}_{0}$, and that can be easily seen to be true whether $\kappa=0$ or $a$. Next we assume that $\mu=a$ and $\nu=b$; then we need to check that

$$
\partial_{\kappa} F_{a b}=-\frac{2}{r^{2}} x_{\kappa} F_{a b}
$$

at $\mathbf{r}_{0}$, and that can be easily verified, too.

We divide the checking of identity (12) at $\mathbf{r}_{0}$ into two cases: (1) one of the indices is zero (easy to check); (2) none of the indices is zero, then the identity becomes

$$
-\left[\gamma_{a b}, \gamma_{c d}\right]=-i \gamma_{a d} \delta_{b c}+i \gamma_{b d} \delta_{a c}-i \gamma_{c a} \delta_{b d}+i \gamma_{c b} \delta_{a d},
$$

which is of course true because $\gamma_{a b}$ are the generators of $\operatorname{so}(D-1)$.

Proof of part (2): We write

$$
r^{2} F_{\lambda \alpha} F_{\lambda \beta}=\frac{r^{2}}{2}\left\{F_{\lambda \alpha}, F_{\lambda \beta}\right\}+\frac{r^{2}}{2}\left[F_{\lambda \alpha}, F_{\lambda \beta}\right]
$$

Using identity (12), we have

$$
r^{2} F_{\lambda \alpha} F_{\lambda \beta}=\frac{r^{2}}{2}\left\{F_{\lambda \alpha}, F_{\lambda \beta}\right\}+i(n-1) F_{\alpha \beta} .
$$

Therefore, by checking at $\mathbf{r}_{0}$, we just need to verify that the identity

$$
\sum_{k}\left\{\gamma_{k i}, \gamma_{k j}\right\}=\frac{\delta_{i j}}{n} \sum_{a, b}\left(\gamma_{a b}\right)^{2}
$$

holds in the irreducible representation $\mathbf{s}^{2 \mu}$ of $\operatorname{so}(2 n)$ whose highest weight is of the form $(|\mu|, \ldots,|\mu|, \mu)$. Since this checking is a bit involved, we do it in the Appendix.

Proof of part (3): We write

$$
r^{2} F_{\lambda \alpha} F_{\lambda \beta}=\frac{r^{2}}{2}\left\{F_{\lambda \alpha}, F_{\lambda \beta}\right\}+\frac{r^{2}}{2}\left[F_{\lambda \alpha}, F_{\lambda \beta}\right]
$$

Using identity (12), we have

$$
r^{2} F_{\lambda \alpha} F_{\lambda \beta}=\frac{r^{2}}{2}\left\{F_{\lambda \alpha}, F_{\lambda \beta}\right\}+i\left(n-\frac{3}{2}\right) F_{\alpha \beta} .
$$

Therefore, by checking at $\mathbf{r}_{0}$, we just need to verify that the identity 


$$
\sum_{k}\left\{\gamma_{k i}, \gamma_{k j}\right\}=(n-1) \delta_{i j}
$$

holds in the spin representation $\mathbf{s}$ of $\mathrm{so}(2 n-1)$, but this is easy to check by using the Clifford algebra.

\section{THE HIDDEN DYNAMICAL SYMMETRY}

To exhibit the dynamical symmetry for our MICZ-Kepler problems, as usual, we introduce the angular momentum tensor

$$
\hat{L}_{\alpha \beta}=-i\left(x_{\alpha} \nabla_{\beta}-x_{\beta} \nabla_{\alpha}\right)+r^{2} F_{\alpha \beta}
$$

and the Runge-Lenz vector

$$
\hat{L}_{\beta}=-\frac{i}{2}\left(\nabla_{\alpha} \hat{L}_{\alpha \beta}+\hat{L}_{\alpha \beta} \nabla_{\alpha}\right)+\frac{x_{\beta}}{r} .
$$

With the help of the identities stated in Lemma 1, a lengthy calculation yields the following commutation relations:

$$
\begin{gathered}
{\left[\hat{L}_{\mu \nu}, \hat{h}\right]=0} \\
{\left[\hat{L}_{\mu \nu}, \hat{L}_{\alpha \beta}\right]=i \delta_{\mu \alpha} \hat{L}_{\nu \beta}-i \delta_{\nu \alpha} \hat{L}_{\mu \beta}-i \delta_{\mu \beta} \hat{L}_{\nu \alpha}+i \delta_{\nu \beta} \hat{L}_{\mu \alpha}} \\
{\left[\hat{L}_{\mu \nu}, \hat{L}_{\lambda}\right]=i \delta_{\mu \lambda} \hat{L}_{\nu}-i \delta_{\nu \lambda} \hat{L}_{\mu}} \\
{\left[\hat{L}_{\mu}, \hat{h}\right]=0} \\
{\left[\hat{L}_{\mu}, \hat{L}_{\nu}\right]=-2 i \hat{h} \hat{L}_{\mu \nu}}
\end{gathered}
$$

provided that either $D$ is odd or $\mu=0, \frac{1}{2}$.

In the remaining part of this section we assume that either $D$ is odd or $\mu=0, \frac{1}{2}$. With this in mind, on the Hilbert space of negative-energy states, we can introduce $\hat{J}_{M N}$ where the capital Latin letters $M$ and $N$ run from 0 to $D$ :

$$
\hat{J}_{M N}= \begin{cases}\hat{L}_{\mu \nu} & \text { if } M=\mu, N=\nu \\ (-2 \hat{h})^{-1 / 2} \hat{L}_{\mu} & \text { if } M=\mu, N=D \\ -(-2 \hat{h})^{-1 / 2} \hat{L}_{\nu} & \text { if } M=D, N=\nu \\ 0 & \text { if } M=N .\end{cases}
$$

Then the commutation relations in Eq. (20) say that a $D$-dimensional MICZ-Kepler problem has a dynamical $\mathrm{SO}(D+1)$ symmetry on the Hilbert space of negative-energy states. Actually, the dynamical symmetry group should be $\operatorname{spin}(D+1)$, rather than $\mathrm{SO}(D+1)$. It is also clear that a $D$-dimensional MICZ-Kepler problem has a dynamical $\operatorname{spin}(D, 1)$ symmetry on the positiveenergy states and a dynamical $\operatorname{spin}(D)+\mathrm{R}^{D}$ symmetry on the zero-energy states.

It also follows from Eq. (20) that $\hat{h}$ must be in the center of the Lie algebra $\operatorname{so}(D+1)$; in fact, it is a function of the quadratic Casimir operator of $\operatorname{so}(D+1)$ : 


$$
\hat{h}=-\frac{1 / 2}{c_{2}[\operatorname{so}(D+1)]+((D-1) / 2)^{2}-\bar{c}_{2}},
$$

where $\bar{c}_{2}$ is the value of $c_{2}[\operatorname{so}(2 n-1)]$ in the spin representation of so(2n-1) if $D=2 n$ and the bundle is $\mathcal{S}$, and is the value of $c_{2}[\operatorname{so}(2 n)]$ in the representation of so(2n) whose highest weight is of the form $(|\mu|, \ldots,|\mu|, \mu)$ if $D=2 n+1$ and the bundle is $\mathcal{S}^{2 \mu}$.

To prove Eq. (21), we first note that $\hat{L}_{\mu}=(-2 \hat{h})^{1 / 2} \hat{J}_{\mu D}$, so $\hat{L}_{\mu} \hat{L}_{\mu}=-2 \hat{h} \Sigma_{\mu} \hat{J}_{\mu D} \hat{J}_{\mu D}$. On the other hand, based on the definition of $L_{\mu}$ given in Eq. (19), a direct computation yields

$$
\hat{L}_{\mu} \hat{L}_{\mu}=1+\left(\frac{1}{2}(D-1)^{2}-2 \bar{c}_{2}+\hat{J}_{\mu \nu} \hat{J}_{\mu \nu}\right) \hat{h}
$$

Therefore,

$$
1+\left(\frac{1}{2}(D-1)^{2}-2 \bar{c}_{2}+\hat{J}_{M N} \hat{J}_{M N}\right) \hat{h}=0
$$

then we have Eq. (21).

It is clear now that in order to determine the spectrum of $\hat{H}$, we just need to find out which irreducible representation of $\operatorname{spin}(D+1)$ enters into the Hilbert space $\mathcal{H}$ of negative-energy states. However, we shall find the discrete spectrum by solving the Schrödinger equation directly and then figure out the decomposition of the Hilbert space of negative-energy states into the irreducible representations of $\operatorname{spin}(D+1)$ via representation theory.

\section{THE SPECTRUM ANALYSIS}

The Schrödinger equation for the stationary states, in terms of the polar coordinates, is

$$
\left(-\frac{1}{2 r^{D-1}} \partial_{r} r^{D-1} \partial_{r}+\frac{c_{2}[\operatorname{so}(D)]-\bar{c}_{2}+\delta_{D}}{2 r^{2}}-\frac{1}{r}\right) \psi=E \psi,
$$

where $E$ is the energy, $c_{2}[\operatorname{so}(D)]=\frac{1}{2} \hat{L}_{\mu \nu} \hat{L}_{\mu \nu}$, and $\delta_{D}$ is equal to $(n-1) \mu$ if $D=2 n$ and is equal to $(n-1)|\mu|+\mu^{2}$ if $D=2 n+1$. Under the action of $\operatorname{spin}(D)$, the Hilbert space of negative-energy states splits into the direct sum of irreducible components. These irreducible components are essentially labeled by a non-negative integer $l$, and we shall be able to see that shortly. On the irreducible component labeled by $l$, the Schrödinger equation becomes an equation for the radial part:

$$
\left(-\frac{1}{2 r^{D-1}} \partial_{r} r^{D-1} \partial_{r}+\frac{c_{2}[l]-\bar{c}_{2}+\delta_{D}}{2 r^{2}}-\frac{1}{r}\right) R_{k l}=E_{k l} R_{k l}
$$

where $c_{2}[l]$ is the value of the quadratic Casimir operator of $\operatorname{so}(D)$ in the irreducible component labeled by $l$, and the additional label $k$ is introduced for the purpose of listing the radial eigenfunctions, just as in the Kepler problem.

Let $E_{k l}=-\frac{1}{2} \lambda_{k l}^{2}$ and $R_{k l}(r)=e^{-\lambda_{k l} r} u_{k l}$; then the preceding radial Schrödinger equation becomes

$$
\left(-\frac{1}{2 r^{D-1}} \partial_{r} r^{D-1} \partial_{r}+\lambda_{k l} \frac{1}{r^{(D-1) / 2}} \partial_{r} r^{(D-1) / 2}+\frac{c_{2}[l]-\bar{c}_{2}+\delta_{D}}{2 r^{2}}-\frac{1}{r}\right) u_{k l}=0 .
$$

Let $y_{k l}=r^{(D-1) / 2} u_{k l}$; then the above equation becomes

$$
\left(\frac{\mathrm{d}^{2}}{\mathrm{~d} r^{2}}-\frac{c_{2}[l]-\bar{c}_{2}+\delta_{D}+(D-1)(D-3) / 4}{r^{2}}+\frac{2}{r}-2 \lambda_{k l} \frac{\mathrm{d}}{\mathrm{d} r}\right) y_{k l}(r)=0 .
$$

Let us assume that $y_{k l}(r) \rightarrow r^{s}$ as $r \rightarrow 0^{+}$, then we must have the following indicial equation: 


$$
s(s-1)=c_{2}[l]-\bar{c}_{2}+\delta_{D}+\frac{(D-1)(D-3)}{4} .
$$

The further analysis is divided into two cases: (1) $D$ is odd and (2) $D$ is even.

\section{A. The odd dimensional cases}

Let $D=2 n+1$. Let $L^{2}\left(\left.\mathcal{S}^{2 \mu}\right|_{S^{2 n}}\right)$ be the $L^{2}$ sections of vector bundle $\mathcal{S}^{2 \mu}$ restricted to the unit sphere $S^{2 n}$. From the representation theory, we know that

$$
L^{2}\left(\left.\mathcal{S}^{2 \mu}\right|_{\left.S^{2 n}\right)}=\oplus_{l \geqslant 0} \mathcal{R}_{l},\right.
$$

where $\mathcal{R}_{l}$ is the irreducible representation space of $\operatorname{spin}(2 n+1)$ having the highest weight equal to $(l+|\mu|,|\mu|, \ldots,|\mu|, \mu)$. It is then clear that the Hilbert spaces of bound states is

$$
\mathcal{H}=\oplus_{l \geqslant 0} \mathscr{H}_{l},
$$

with $\mathscr{H}_{l}$ being a subspace of $L^{2}\left(\mathrm{R}_{+}, r^{2 n} \mathrm{~d} r\right) \otimes \mathcal{R}_{l}$. Here $L^{2}\left(\mathbb{R}_{+}, r^{2 n} \mathrm{~d} r\right)$ is the $L^{2}$ space of complexvalued functions on half-line $\mathbb{R}_{+}$with measure $r^{2 n} \mathrm{~d} r$.

The value of the quadratic Casimir operator of $\operatorname{so}(2 n)$ on representation $\mathbf{s}^{2 \mu}$ is

$$
\bar{c}_{2}=n \mu^{2}+n(n-1)|\mu| .
$$

The value of the quadratic Casimir operator of $\operatorname{so}(2 n+1)$ on $\mathcal{R}_{l}$ is

$$
c_{2}[l]=l^{2}+2 l\left(n+|\mu|-\frac{1}{2}\right)+n \mu^{2}+n^{2}|\mu| .
$$

Plugging the values for $\bar{c}_{2}$ and $c_{2}[l]$ into Eq. (26), we get

$$
s(s-1)=(l+n+|\mu|)(l+n+|\mu|-1) .
$$

Therefore, $s=l+n+|\mu|$ or $s=1-l-n-|\mu|$. The solution $s=1-l-n-|\mu|$ must be rejected; otherwise, the wave functions cannot be square integrable near $r=0$. Just as in solving the hydrogen atom problem, with $s=l+n+|\mu|$, we continue the analysis by setting

$$
y_{k l}=r^{s} \sum_{m=0}^{\infty} a_{m} r^{m},
$$

with $a_{0}=1$, and then get the recursive relation: for $m \geqslant 1$, one has

$$
a_{m}((m+s)(m+s-1)-s(s-1))=\left(1-\lambda_{k l}(m+s-1)\right) a_{m-1} .
$$

As it has been demonstrated in Ref. 11 the power series solution must be a polynomial solution; otherwise, the wave function will not be square integrable for $r$ near infinity. Therefore, we must have

$$
\lambda_{k l}=\frac{1}{k+s-1}=\frac{1}{k+l+n+|\mu|-1}
$$

and that leads to the energy spectrum

$$
E_{k l}=-\frac{1 / 2}{(k+l+n+|\mu|-1)^{2}},
$$

where $k$ must be a positive integer, and an orthogonal decomposition 


$$
\mathscr{H}_{l}=\oplus_{k=1}^{\infty} \mathcal{H}_{k l},
$$

with each of $\mathcal{H}_{k l}$ being isomorphic to $\mathcal{R}_{l}$ as $\operatorname{spin}(2 n+1)$ modules. Therefore, in view of Eq. (28), we have an orthogonal decomposition of $\mathcal{H}$ into energy eigenstates:

$$
\mathcal{H}=\oplus_{I=0}^{\infty} \mathcal{H}_{I},
$$

where

$$
\mathcal{H}_{I}=\oplus_{k+l=I+1} \mathcal{H}_{k l} .
$$

Since the linear action of $\operatorname{spin}(2 n+2)$ on $\mathcal{H}$ commutes with the Hamiltonian, this linear action must leave the energy eigenstates $\mathcal{H}_{I}$ invariant. On the other hand, from representation theory, as a $\operatorname{spin}(2 n+1)$ module, being isomorphic to

$$
\oplus_{l=0}^{I} \mathcal{R}_{l}
$$

$\mathcal{H}_{I}$ must be the irreducible representation of $\operatorname{spin}(2 n+2)$ whose highest weight must be (I $+|\mu|,|\mu|, \ldots,|\mu|, \pm|\mu|)$. As a consistency check, one can see that Eq. (21) yields

$$
E_{I}=-\frac{1 / 2}{(I+n+|\mu|)^{2}}
$$

on such representation, in complete agreement with Eq. (30) because $I=k+l-1$. One can show that, as a $\operatorname{spin}(2 n+2)$ module, $\mathcal{H}_{I}$ has the highest weight equal to $(I+|\mu|,|\mu|, \ldots,|\mu|, \mu)$.

In summary, the energy spectrum is

$$
E_{I}=-\frac{1 / 2}{(I+n+|\mu|)^{2}},
$$

where $I=0,1,2, \ldots$, and $\mathcal{H}$ furnishes a representation for $\operatorname{spin}(2 n+2)$ and has the following decomposition into energy eigenstates:

$$
\mathcal{H}=\oplus_{I=0}^{\infty} \mathcal{H}_{I},
$$

where $\mathcal{H}_{I}$ is the irreducible component of $\mathcal{H}$ whose highest weight is $(I+|\mu|,|\mu|, \ldots,|\mu|, \mu)$.

\section{B. The even dimensional cases}

Let $D=2 n$. Let $L^{2}\left(\left.\mathcal{S}^{2 \mu}\right|_{S^{2 n-1}}\right)$ be the $L^{2}$ sections of vector bundle $\mathcal{S}^{2 \mu}$ restricted to the unit sphere $S^{2 n-1}$. From the representation theory, we know that

$$
L^{2}\left(\left.\mathcal{S}^{2 \mu}\right|_{S^{2 n-1}}\right)=\oplus_{l \geqslant 0}\left(\mathcal{R}_{l^{+}} \oplus \mathcal{R}_{l^{-}}\right),
$$

where $\mathcal{R}_{l^{ \pm}}$is the irreducible representation space of $\operatorname{spin}(2 n)$ having the highest weight equal to $(l+\mu, \mu, \ldots, \mu, \pm \mu)$. It is then clear that the Hilbert spaces of bound states is

$$
\mathcal{H}=\oplus_{l \geqslant 0}\left(\mathscr{H}_{l^{+}} \oplus \mathscr{H}_{l^{+}}\right),
$$

with $\mathscr{H}_{l^{ \pm}}$being a subspace of $L^{2}\left(\mathbb{R}_{+}, r^{2 n-1} \mathrm{~d} r\right) \otimes \mathcal{R}_{l^{ \pm}}$. Here $L^{2}\left(\mathbb{R}_{+}, r^{2 n-1} \mathrm{~d} r\right)$ is the $L^{2}$ space of complex-valued functions on half-line $\mathbb{R}_{+}$with measure $r^{2 n-1} \mathrm{~d} r$.

The value of the quadratic Casimir operator of $\operatorname{so}(2 n-1)$ on representation $\mathbf{s}^{2 \mu}$ is

$$
\bar{c}_{2}=(n-1) \mu^{2}+(n-1)^{2} \mu .
$$

The value of the quadratic Casimir operator of $\operatorname{so}(2 n)$ on $\mathcal{R}_{l^{ \pm}}$is

$$
c_{2}[l]=l^{2}+2 l(n+\mu-1)+n \mu^{2}+\left(n^{2}-n\right) \mu .
$$

Plugging the values for $\bar{c}_{2}$ and $c_{2}[l]$ into Eq. (26), we get 


$$
s(s-1)=\left(l+n+\mu-\frac{1}{2}\right)\left(l+n+\mu-\frac{3}{2}\right) .
$$

Therefore, $s=l+n+\mu-\frac{1}{2}$ or $s=\frac{3}{2}-l-n-\mu$. The solution $s=\frac{3}{2}-l-n-\mu$ must be rejected; otherwise, the wave functions cannot be square integrable near $r=0$. Just as in solving the hydrogen atom problem, with $s=l+n+\mu-\frac{1}{2}$, we continue the analysis by setting

$$
y_{k l}=r^{s} \sum_{m=0}^{\infty} a_{m} r^{m},
$$

with $a_{0}=1$, and then get the recursive relation: for $m \geqslant 1$, one has

$$
a_{m}((m+s)(m+s-1)-s(s-1))=\left(1-\lambda_{k l}(m+s-1)\right) a_{m-1} .
$$

As it has been demonstrated in Ref. 11, the power series solution must be a polynomial solution; otherwise, the wave function will not be square integrable for $r$ near infinity. Therefore, we must have

$$
\lambda_{k l}=\frac{1}{k+s-1}=\frac{1}{k+l+n+\mu-3 / 2}
$$

and that leads to the energy spectrum

$$
E_{k l}=-\frac{1 / 2}{(k+l+n+\mu-3 / 2)^{2}},
$$

where $k$ must be a positive integer, and an orthogonal decomposition

$$
\mathscr{H}_{l^{ \pm}}=\oplus_{k=1}^{\infty} \mathcal{H}_{k l^{ \pm}},
$$

with each of $\mathcal{H}_{k l^{ \pm}}$being isomorphic to $\mathcal{R}_{l^{ \pm}}$as $\operatorname{spin}(2 n)$ modules. Therefore, in view of Eq. (36), we have an orthogonal decomposition of $\mathcal{H}$ into energy eigenstates:

$$
\mathcal{H}=\oplus_{I=0}^{\infty} \mathcal{H}_{I},
$$

where

$$
\mathcal{H}_{I}=\oplus_{k+l=I+1}\left(\mathcal{H}_{k l^{+}} \oplus \mathcal{H}_{k l^{-}}\right) .
$$

Note that $\mathcal{H}_{I}$ is isomorphic to

$$
\oplus_{l=0}^{I}\left(\mathcal{R}_{l^{+}} \oplus \mathcal{R}_{l^{-}}\right),
$$

as a $\operatorname{spin}(2 n)$ module. From the representation theory, the manifest $\operatorname{spin}(2 n)$ linear action on $\mathcal{H}_{I}$ can be extended to a linear action of $\operatorname{spin}(2 n+1)$ such that $\mathcal{H}_{I}$ is the irreducible representation of $\operatorname{spin}(2 n+1)$ whose highest weight is $(I+\mu, \mu, \ldots, \mu)$ and Eq. (21) is valid. As a consistency check, one can see that Eq. (21) yields

$$
E_{I}=-\frac{1 / 2}{(I+n+\mu-1 / 2)^{2}}
$$

on such representation, in complete agreement with Eq. (38) because $I=k+l-1$. However, the components of the Runge-Lenz vector defined in Eq. (19) are not symmetry generators.

In summary, the energy spectrum is 


$$
E_{I}=-\frac{1 / 2}{(I+n+\mu-1 / 2)^{2}},
$$

where $I=0,1,2, \ldots$, and $\mathcal{H}$ furnishes a representation for $\operatorname{spin}(2 n+1)$ and has the following decomposition into energy eigenstates:

$$
\mathcal{H}=\oplus_{I=0}^{\infty} \mathcal{H}_{I},
$$

where $\mathcal{H}_{I}$ is the irreducible component of $\mathcal{H}$ whose highest weight is $(I+\mu, \mu, \ldots, \mu)$.

\section{ACKNOWLEDGMENTS}

I would like to thank SiXia Yu for a conversation on the Kepler problem. This work is supported by the Hong Kong Research Grants Council under RGC Project No. 602504.

\section{APPENDIX: PROOF OF THE REMAINING PART OF LEMMA 1}

To prove identity (16), we first note that we just need to prove that identities

$$
\sum_{k}\left(\gamma_{1, k}\right)^{2}=\frac{1}{n} c_{2}
$$

and

$$
\sum_{k}\left\{\gamma_{1, k}, \gamma_{2, k}\right\}=0
$$

hold in the representation $\mathbf{s}_{+}^{2 \mu}$ for any non-negative integer $\mu$.

To continue, a digression on Lie algebra so $(2 n)$ is needed. Recall that the root space of $\operatorname{so}(2 n)$ is $\mathbb{R}^{n}$. Let $e^{i}$ be the vector in $\mathbb{R}^{n}$ whose $i$ th entry is 1 and all other entries are zero. The positive roots are $e^{i} \pm e^{j}$ with $1 \leqslant i<j \leqslant n$. The simple roots are $\alpha^{i}=e^{i}-e^{i+1}, i=1$ to $n-1$ and $\alpha^{n}=e^{n-1}+e^{n}$. For the Cartan basis, we make the following choice: The commuting generators in the Cartan subalgebra are taken to be

$$
H_{j}=\gamma_{2 j-1,2 j} \quad j=1 \quad \text { to } n,
$$

and the $E$ generators are taken to be

$$
E_{\eta e^{j}+\eta^{\prime} e^{k}}=\frac{1}{2}\left(\gamma_{2 j-1,2 k-1}+i \eta \gamma_{2 j, 2 k-1}+i \eta^{\prime} \gamma_{2 j-1,2 k}-\eta \eta^{\prime} \gamma_{2 j, 2 k}\right) \text {, }
$$

where $j<k$ and $\eta, \eta^{\prime} \in\{1,-1\}$. Note that the fact that $\left[E_{\alpha}, E_{\beta}\right]=0$ if $\alpha+\beta$ is neither a root nor zero is frequently used in all subsequent calculations. All of these are standard materials taken from a textbook such as Ref. 12

Let

$$
\begin{gathered}
\mathcal{O}=\sum_{n \geqslant i \geqslant 2} E_{-e^{1}-e^{i}} E_{-e^{1}+e^{i}}, \\
\mathcal{O}_{1}=H_{1}^{2}+\frac{1}{2} \sum_{n \geqslant i \geqslant 2}\left(\left\{E_{-e^{1}-e^{i}}, E_{e^{1}+e^{i}}\right\}+\left\{E_{-e^{1}+e^{i}}, E_{e^{1}-e^{i}}\right\}\right) .
\end{gathered}
$$

By simple computations, we have

$$
\sum_{k}\left(\gamma_{1, k}\right)^{2}=\mathcal{O}_{1}+\mathcal{O}^{\dagger}+\mathcal{O}
$$




$$
\sum_{k}\left\{\gamma_{1, k}, \gamma_{2, k}\right\}=\frac{2}{i}\left(\mathcal{O}^{\dagger}-\mathcal{O}\right)
$$

It is then clear from the above calculations that identities (A1) and (A2) are valid modulo the following claim:

Claim 1: Let $|\Lambda\rangle$ be an element of the so(2n) module $\mathbf{s}_{+}^{2 \mu}$. Then

$$
\begin{gathered}
\mathcal{O}|\Lambda\rangle=0, \\
\mathcal{O}^{\dagger}|\Lambda\rangle=0, \\
\mathcal{O}_{1}|\Lambda\rangle=\mu(n+\mu-1)|\Lambda\rangle \\
=\frac{1}{n} c_{2}|\Lambda\rangle .
\end{gathered}
$$

Proof of the claim: We first remark that, among the four equalities in the claim, we just need to prove the first and the third, that is because the second is a consequence of the first and the last is true because $c_{2}=n \mu(n+\mu-1)$. We also remark that we may assume that $|\Lambda\rangle$ is a state created from $|\mu, \ldots, \mu\rangle$ by applying a bunch of lowing operators of the form $E_{-\alpha} j$, that is because a general state is always a linear combination of states of this kind.

Next, we observe that

$$
E_{-e^{1}+e^{i}}|\mu, \cdots, \mu\rangle=0
$$

consequently, $\mathcal{O}|\mu, \ldots, \mu\rangle=0$. This observation can be shown by the following trick: Let $e$

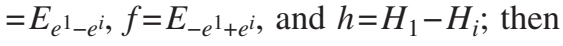

$$
\begin{gathered}
{[h, e]=2 e,} \\
{[h, f]=-2 f,} \\
{[e, f]=h .}
\end{gathered}
$$

That is, $\{e, f, h\}$ forms the standard Cartan basis for su(2). It follows from the following computation:

$$
\| f|\mu, \cdots, \mu\rangle \|^{2}=\langle\mu, \cdots, \mu|e f| \mu, \cdots, \mu\rangle=\langle\mu, \cdots, \mu|h+f e| \mu, \cdots, \mu\rangle=0
$$

that $f|\mu, \ldots, \mu\rangle=0$. Moreover, when $|\Lambda\rangle=|\mu, \ldots, \mu\rangle$, the third identity of the claim is just the consequence of a direct computation. Therefore, the claim is true when $|\Lambda\rangle=|\mu, \ldots, \mu\rangle$.

Finally, we need to reduce the general case to the special case discussed in the previous paragraph. Combining the computational fact that

$$
\left[E_{-e^{1}+e^{j}}, E_{-e^{j}+e^{j+1}}\right]=-i E_{-e^{1}+e^{j+1}}, \quad\left[E_{-e^{1}-e^{j+1}}, E_{-e^{j}+e^{j+1}}\right]=i E_{-e^{1}-e^{j}}
$$

(where $1<j<n$ ) and the computational fact that

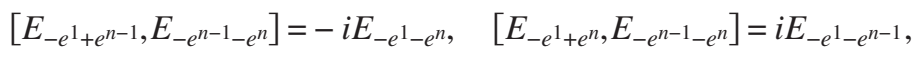

one can show that $\left[\mathcal{O}, E_{-\alpha j}\right]=0$ for any $1 \leqslant j \leqslant n$. Since $|\Lambda\rangle$ can be assumed to be a state created from $|\mu, \ldots, \mu\rangle$ by applying a bunch of lowing operators of the form $E_{-\alpha}$, in view of the fact that $\mathcal{O}|\mu, \ldots, \mu\rangle=0$, we have $\mathcal{O}|\Lambda\rangle=0$.

The reduction to the special case for the third identity is a bit involved. The key is to introduce a series of operators: 


$$
\begin{gathered}
\mathcal{O}_{2}=-2\left[\mathcal{O}_{1}, E_{-\alpha^{1}}\right], \\
\mathcal{O}_{k}=i\left[\mathcal{O}_{k-1}, E_{-\alpha^{k-1}}\right] \quad \text { for } 3 \leqslant k \leqslant n, \\
\mathcal{O}^{n-2}=-i\left[\mathcal{O}_{n}, E_{-\alpha^{n}}\right], \\
\mathcal{O}^{k-1}=-i\left[\mathcal{O}^{k}, E_{-\alpha^{k}}\right] \quad \text { for } 1 \leqslant k \leqslant n-2,
\end{gathered}
$$

and then observe that

$$
\begin{gathered}
{\left[\mathcal{O}_{k}, E_{-\alpha^{j}}\right]=0 \quad \text { if } 1 \leqslant j \leqslant n, 1 \leqslant k \leqslant n, \quad \text { and } j \neq k} \\
{\left[\mathcal{O}^{k}, E_{-\alpha^{j}}\right]=0 \text { if } 1 \leqslant j \leqslant n, 1 \leqslant k \leqslant n-2, \quad \text { and } j \neq k} \\
\qquad \mathcal{O}^{0}=4 i \mathcal{O}, \\
\mathcal{O}_{k}|\mu, \cdots, \mu\rangle=0 \quad \text { for } 2 \leqslant k \leqslant n, \\
\mathcal{O}^{k}|\mu, \cdots, \mu\rangle=0 \quad \text { for } 1 \leqslant k \leqslant n-2 .
\end{gathered}
$$

With the help of the above equations starting from Eq. (A15) and ending at Eq. (A16) plus the proven fact that $\mathcal{O}|\Lambda\rangle=0$, an induction argument finishes the proof. Here the induction is done on the number of lowing operators of the form $E_{-\alpha^{j}}$ which are used to create state $|\Lambda\rangle$.

We end this Appendix with the following conjecture: identity (16) holds if and only if when the representation is a Young power of $\mathbf{s}_{+}$or $\mathbf{s}_{-}$. For our purpose, we have just proven the "if" part in this Appendix.

${ }^{1}$ B. Cordani, The Kepler Problem: Group Theoretical Aspects, Regularization and Quantization, with Application to the Study of Perturbation (Birkhäuser, Basel, 2002).

${ }^{2}$ H. McIntosh and A. Cisneros, J. Math. Phys. 11, 896 (1970).

${ }^{3}$ D. Zwanziger, Phys. Rev. 176, 1480 (1968).

${ }^{4}$ G. W. Meng, e-print math-ph/0409051.

${ }^{5}$ T. Iwai, J. Geom. Phys. 7, 507 (1990).

${ }^{6}$ C. N. Yang, J. Math. Phys. 19, 320 (1978).

${ }^{7}$ G. W. Meng, J. Phys. A 36, 9415 (2003).

${ }^{8}$ D. Simms, Geometric Quantization of the Energy Levels in Kepler Problem, In Symposia Mathematica (INDAM, Rome, 1974), Vol. 14, pp. 125-138.

${ }^{9}$ I. Mladenov and V. Tsanov, J. Geom. Phys. 2, 17 (1985).

${ }^{10}$ I. Mladenov and V. Tsanov, J. Phys. A 20, 5865 (1987).

${ }^{11}$ C. Cohen-Tannoudji, B. Diu, and F. Laloë, Quantum Mechanics (Wiley-Interscience, New York, 1977), Vol. I.

${ }^{12}$ H. Georgi, Lie Algebras in Particle Physics (Benjamin, London, 1982). 